\title{
FASTER-THAN-NYQUIST SPATIOTEMPORAL SYMBOL-LEVEL PRECODING IN THE DOWNLINK OF MULTIUSER MISO CHANNELS
}

\author{
Maha Alodeh, Danilo Spano, Symeon Chatzinotas, and Björn Ottersten \\ Interdisciplinary Centre for Security Reliability and Trust, University of Luxembourg \\ \{maha.alodeh, danilo.spano, symeon.chatzinotas, bjorn.ottersten\}@ uni.lu
}

\begin{abstract}
This paper investigates the problem of interference among the simultaneous multiuser transmissions in the downlink of multiple antennas systems. Symbol-level precoding (SLP) is a promising technique which has recently demonstrated large performance gains over the conventional block-level techniques. These gains can be translated in lower power requirements, improved energy efficiency, lower peak to average power ratio and resilience to non-linearities. However, previous works have not exploited the full potentials of SLP as it was only used to exploit multiuser interference spatially. In this paper, we extend this concept by using Faster-than-Nyquist (FTN) signaling and employing SLP to manage both multi-user and inter-symbol interference (ISI). We consider the aforementioned paradigm in the context of Massive multiple-input multiple-output (MIMO) systems, where the number of transmit antennas is usually an order of magnitude larger than the number of served users. In this rich degrees of freedom (DoF) environment, we show that FTN SLP can double the effective user rates while improving the energy efficiency.
\end{abstract}

Index Terms - Faster-than-Nyquist, inter-symbol interference, multiuser MIMO, spatiotemporal precoding, symbol-level.

\section{INTRODUCTION}

Faster-than-Nyquist signaling promises higher spectral efficiencies by introducing controlled intersymbol interference at the transmitter. In FTN, data streams are sampled and transmitted at a fraction of symbol period which improves the data rates at the expense of increased receiver complexity to combat intersymbol interference (ISI) [1]-[7]. Mazo showed that symbols carried by sinc pulses can be transmitted at a higher rate than that dictated by Nyquist without sacrificing the system bit error rate[1]. In [2], the lower and upper bounds of the information rates was investigated using root raised cosine pulses for binary, quaternary and octal FTN schemes. They proved that in many cases, FTN gives higher information rate than the Nyquist case due to the benefit of using the excess pulse bandwidth, which is the small bandwidth added by a pulse that decays slower than a sinc pulse and satisfies the Nyquist criterion. A multicarrier FTN was proposed in [4] where Mazo's limit is applied over two dimensions, time and frequency. It was shown that the single carrier limit $0.825 T$ decreases when it is applied over two dimen-

This work is supported by H2020 Project SANSA (Shared Access Terrestrial-Satellite Backhaul Network enabled by Smart Antennas) and Fond National de la Recherche Luxembourg (FNR) projects, Spectrum Management and Interference Mitigation in Cognitive Radio Satellite Networks (SeMiGod) and SATellite SEnsor NeTworks for spectrum monitoring (SATSENT). sions as compared to the time domain only, so that higher data rate can be achieved.

In parallel, MIMO systems have attracted significant research interest during the last decade due to various advantages they promise, enhanced capacity, energy efficiency, etc. It has been recently shown that the employment of an excess of antennas at the BS (very large MIMO) offers unprecedented array and multiplexing gains both in the uplink and in the downlink [8]-[11]. On the other hand, symbollevel precoding (SLP) is a new paradigm that can tackle the interference into a constructive fashion if we process the transmitted data frame at symbol level [13]-[17]. In [12]-[13][16], the optimal SLP strategy for the minimization of the total transmit power, was given under user quality of service (QoS) guarantees at each user. In further extensions, SLP design is generalized under the assumption that the received MPSK symbol can reside in a relaxed region in order to be correctly detected [15].

In this paper, we aim at increasing the energy efficiency and the effective rate of the multiuser MISO by inducing intentional ISI using FTN in time domain. As a result, this setup introduces spatiotemporal interference to have spatial and temporal interference among the users' data streams. The channel becomes more interference limited since each transmitted data symbol faces interference from the other users' symbols and from the previous and the following symbols. We propose symbol-level precoding that can exploit the spatial and temporal interference to glean the benefits in both domains. It should be highlighted thar SLP techniques simplify the receiver architecture for FTN based system since the ISI is already handled at the transmitter and there is no need for complex processing at the user terminal.

Notation: We use boldface upper and lower case letters for matrices and column vectors, respectively. $(\cdot)^{H},(\cdot)^{*}$ stand for Hermitian transpose and conjugate of $(\cdot) . \mathbb{E}(\cdot)$ and $\|\cdot\|$ denote the statistical expectation and the Euclidean norm. $\angle(\cdot),|\cdot|$ are the angle and magnitude of $(\cdot)$ respectively. $\mathcal{R}(\cdot), \mathcal{I}(\cdot)$ are the real and the imaginary part of $(\cdot), i$ indicates the complex part of the number. $\odot, \otimes$ denotes the Hadamard and Kronecker products respectively. Finally, $\mathbf{1}_{a \times b}$ and $\mathbf{I}_{a}$ denote the matrix of all ones of size $a \times b$ and identity matrix of size $a \times a$.

\section{SYSTEM MODEL}

Let us assume that system communicates $K$ independent messages to $K$ single-antenna users through $N$ transmit antennas. Each data stream is divided in blocks of $S$ symbols. The channel is assumed to be quasi-static block fading, namely it remains constant for each block. In this context, $\mathbf{S}=\left[\mathbf{s}_{1} \ldots \mathbf{s}_{K}\right]^{T}$ is an $K \times S$ matrix aggregating the $K \times 1$ input symbol vectors for each symbol slot. Similarly, $\mathbf{D}$ is a $N \times S$ matrix representing the input signal vectors to 
the $N$ transmit antennas for each symbol slot. Each input signal has to undergo pulse shaping before transmission. The analog waveform $\alpha(t)$ is formally defined through its discrete samples $\alpha[i T]$, where $T$ is the symbol period. Assuming a unit-power symmetric pulse centered at zero with duration $2 \eta T$, we can state that $\alpha[i T]=\alpha[-i T]$ and $\alpha[i T] \approx 0$ for $i<-\eta$ or $i>\eta^{1}$.

Due to the pulse memory, the $s$ th transmitted signal by the $m$ th antenna can be written as:

$$
[\mathbf{X}]_{m s}=\sum_{i=-\eta}^{\eta} \alpha[i T][\mathbf{D}]_{m i},
$$

where $\mathbf{X}$ is a $N \times S$ matrix representing the output signal vectors to the $N$ transmit antennas for each symbol slot. Now let us define a symmetric $S \times S$ Toeplitz matrix A whose first row is defined as $\mathbf{a}=[\alpha[0] \alpha[T] \ldots \alpha[\eta T] 0 \ldots 0]$. As a result, the pulse shaping process can be expressed as a linear multiplication $\mathbf{X}=\mathbf{D A}$.

According to the well-known multiuser MISO channel model, the received symbols at the users can be written in matrix form as:

$$
\mathbf{Y}=\mathbf{H X}+\mathbf{Z}=\mathbf{H D A}+\mathbf{Z}
$$

where $\mathbf{Y}$ is a $K \times S$ matrix representing the received signals at the $K$ users for each symbol slot, $\mathbf{H}=\left[\mathbf{h}_{1} \ldots \mathbf{h}_{K}\right]^{T}$ is a $K \times N$ matrix representing the spatial channels among $M$ antennas and $K$ users and $\mathbf{Z}$ is a matrix representing the Additive White Gaussian Noise $(\mathrm{AWGN})^{2}$. By vectorizing over the time dimension (rows first) for a single block, we can further expand the above model into a simpler form, the vector representing the transmitted signals from all $M$ antennas for all $S$ symbols can be formulated as:

$$
\mathbf{x}=\operatorname{vec}\left(\mathbf{X}^{T}\right)=\left(\mathbf{I}_{K} \otimes \mathbf{A}^{T}\right) \mathbf{d},
$$

and the received signal can be expressed in a vector form as:

$$
\mathbf{y}=\underbrace{\left(\mathbf{H} \otimes \mathbf{A}^{T}\right)}_{\mathbf{G}} \mathbf{d}+\mathbf{z},
$$

where $\mathbf{y}=\operatorname{vec}\left(\mathbf{Y}^{T}\right)$ is a $K S \times 1$ vector representing the received signals at all $K$ users for all $S$ symbols, $\mathbf{d}$ is a $N S \times 1$ vector representing the input signals before pulse shaping at all $N$ antennas for all $S$ symbols and $\mathbf{z}$ is a $K S \times 1$ vector representing the AWGN. $\mathbf{G}$ is a $K S \times N S$ matrix representing the spatiotemporal channel matrix and $\mathbf{z}$ is a $K S \times 1$ vector representing the AWGN at all $K$ users and all $S$ symbols.

\subsection{Faster-than-Nyquist}

FTN signaling manages to pack more information in time domain, see Fig. 1. In the system model definition, we have not made any assumptions on the symbol-rate so far. It can be easily deduced that if Nyquist signaling with symbol period $T_{\text {ny }}$ is used in combinations with sinc pulses, $\alpha[i T]=0, \forall i \neq 0$ are zero and the Toeplitz matrix reduces to a scaled identity $\mathbf{A}=\alpha[0] \mathbf{I}_{S}$. As a result, the output signal after pulse shaping can be straightforwardly expressed as: $\mathbf{X}=\alpha[0] \mathbf{D}$.

Now, let us assume that we apply a signaling acceleration factor $\tau \leq 1$, so that the effective symbol period is $T=\tau T_{\mathrm{ny}}$. It can be

\footnotetext{
${ }^{1}$ For infinite pulses, $\eta$ is defined by the time required so that the pulse decades below a sufficiently low level so that the ISI can be considered negligible.

${ }^{2}$ In this paper, we focus on real channels, although it can be straightforwardly applied to complex channels. According to [19], the complex channel model can be reformulated into a real one.
}
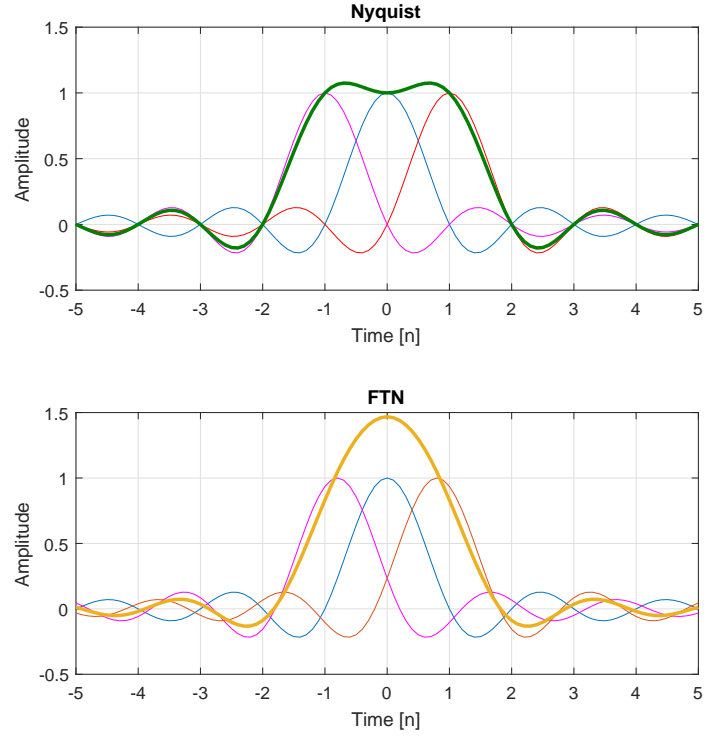

Fig. 1. Nyquist vs FTN in time domain.

easily seen that the higher the acceleration the larger is the number of non-zero values in the $\mathbf{A}$ matrix. This can be easily explained by the fact that more pulses are packed in the time domain. Furthermore, as the total duration of the individual pulse remains constant, the value of $\eta$ increases as $\tau$ decreases [2]. For analytical simplicity, in this paper we adopt a truncated Gaussian pulse shaping, so that the pulse duration is not infinite and the $\alpha$ values decrease monotonically.

\section{FTN SYMBOL LEVEL PRECODING}

\subsection{Precoder Design}

Previous SLP solutions aimed at exploiting only the spatial domain interference [13]-[18]. Using FTN signaling creates temporal correlation among the symbols (ISI) in time domain which can be utilized to design spatiotemporal precoding. This type of precoding is capable of exploiting the interference in time and spatial domain. The new optimization should take into the account the multiuser interference and ISI. In this work, we aim at minimizing total power of the transmitted waveforms under per user quality of service (QoS) constraint. This QoS constraint is selected based on the required modulation and symbol error rate (SER) and it is predefined and fixed for the whole frame for each user. Morevoer, users can have different QoS requirements. The optimization can be written in a matrix form as:

$$
\begin{array}{r}
\mathbf{D}(\mathbf{S}, \mathbf{H}, \mathbf{A}, \gamma)=\frac{\min _{\mathbf{D}}\|\mathbf{D A}\|_{F}^{2}}{S} \\
\mathbf{H D A} \unlhd \mathbf{Q} \odot \mathbf{S},
\end{array}
$$

where $\mathbf{Q}=\sqrt{\boldsymbol{\gamma}} \otimes \mathbf{1}_{1 \times S}, \boldsymbol{\gamma}=\left[\gamma_{1}, \ldots, \gamma_{K}\right]^{T}$ are the SNR targets for each user and $\unlhd$ is element wise operator that guarantee that each received symbol is in its correct detection region [16]. Using the vector representation as in (3)-(4), the optimization in (5) can be 
reformulated $\mathrm{as}^{3}$ :

$$
\begin{aligned}
& \mathbf{d}(\mathbf{s}, \mathbf{H}, \mathbf{A}, \gamma)=\min _{\mathbf{d}}\|\mathbf{x}\|^{2} \\
& \text { s.t. } \quad \mathbf{G}_{j} \mathbf{d} \unlhd \sqrt{\gamma_{j}} \mathbf{s}_{j} \forall j \in K,
\end{aligned}
$$

where $\mathbf{G}_{j}=\left[\mathbf{g}_{j}^{T}[1], \ldots, \mathbf{g}_{j}^{T}[S]\right]^{T}$ denotes the $S \times N S$ spatiotemporal channel matrix for the user $j$, and $\mathbf{s}_{j}$ is $S \times 1$ vector representing the data symbol for user $j$ over $S$ symbols. It should be noted that this optimization problem is solved at symbol-level without ignoring the ISI from the next symbols. The effect of ISI is captured by the matrix G. By further manipulating the optimization in (6), the problem can be written as:

$$
\begin{array}{ll}
\mathbf{d} & (\mathbf{s}, \mathbf{H}, \mathbf{A}, \boldsymbol{\gamma})=\min _{\mathbf{d}}\left\|\left(\mathbf{I} \otimes \mathbf{A}^{T}\right) \mathbf{d}\right\|^{2}, \\
\text { s.t. } & \left\{\begin{array}{l}
\mathcal{C}_{1}:\left\|\mathbf{g}_{j}[n] \mathbf{d}\right\|^{2} \unlhd \kappa_{j}^{2}[n] \gamma_{j} \sigma^{2} \\
\mathcal{C}_{2}: \angle \mathbf{g}_{j}[n] \mathbf{d}=\angle s_{j}[n], \forall j \in K, \forall n \in S
\end{array}\right.
\end{array}
$$

where $\kappa_{j}$ denotes the factor $\kappa_{j}=\left|s_{j}\right| / \sqrt{\mathbb{E}_{\mathcal{D}}\left[\left|s_{j}\right|^{2}\right]}$ changes on a symbol-basis and adjusts the long-term SINR based on the amplitude of the desired symbol [16]. The first two sets of constraints $\mathcal{C}_{1}$ and $\mathcal{C}_{2}$ grant the reception of the data symbols with certain signal to noise ratio (SNR) level.

\subsubsection{Detailed Optimization For Circular Modulation}

For any circular modulation (MPSK, APSK), the optimization can be expanded as following:

$$
\begin{array}{ll}
\mathbf{d} \quad(\mathbf{s}, \mathbf{H}, \mathbf{A}, \boldsymbol{\gamma})=\min _{\mathbf{d}}\left\|\left(\mathbf{I} \otimes \mathbf{A}^{T}\right) \mathbf{d}\right\|^{2}, \\
\text { s.t. } \quad\left\{\begin{array}{l}
\mathcal{C}_{1}: \mathcal{R}\left\{\mathbf{g}_{j}[n] \mathbf{d}\right\} \unlhd \sqrt{\gamma_{j}} \sigma \mathcal{R}\left\{s_{j}[n]\right\} \\
\mathcal{C}_{2}: \mathcal{I}\left\{\mathbf{g}_{j}[n] \mathbf{d}\right\} \unlhd \sqrt{\gamma_{j}} \sigma \mathcal{I}\left\{s_{j}[n]\right\} \forall j \in K, \forall n \in S(8) \\
\mathcal{C}_{3}: \mathcal{I}\left\{\mathbf{g}_{j}[n] \mathbf{d}\right\}-a_{j}[n] \mathcal{R}\left\{\mathbf{g}_{j}[n] \mathbf{d}\right\}=0,
\end{array}\right.
\end{array}
$$

where

$$
\begin{aligned}
\mathcal{R}\left\{\mathbf{g}_{j}[n] \mathbf{d}\right\} & =\frac{\mathbf{g}_{j}[n] \mathbf{d}+\mathbf{d}^{H} \mathbf{g}_{j}^{H}[n]}{2}, \\
\mathcal{I}\left\{\mathbf{g}_{j}[n] \mathbf{d}\right\} & =\frac{\mathbf{g}_{j}[n] \mathbf{d}-\mathbf{d}^{H} \mathbf{g}_{j}^{H}[n]}{2 i} \\
a_{j}[n] & =\tan \left(\angle s_{j}[n]\right) .
\end{aligned}
$$

$\mathcal{C}_{1}, \mathcal{C}_{2}$ and $\mathcal{C}_{3}$ can be formulated to guarantee that the received signal lies in the correct detection region, which depends on the data symbols. A detailed formulation for $\mathcal{C}_{1}, \mathcal{C}_{2}$ can be expressed as

- For the inner-constellation symbols, the constraints $\mathcal{C}_{1}, \mathcal{C}_{2}$ should guarantee that the received signals achieve the exact constellation point. The constraints can be written as:

$$
\begin{array}{ll}
\mathcal{C}_{1}: & \mathcal{R}\left\{\mathbf{g}_{j}[n] \mathbf{d}\right\}=\sigma \sqrt{\gamma_{j}} \mathcal{R}\left\{s_{j}[n]\right\} \\
\mathcal{C}_{2}: & \mathcal{I}\left\{\mathbf{g}_{j}[n] \mathbf{d}\right\}=\sigma \sqrt{\gamma_{j}} \mathcal{I}\left\{s_{j}[n]\right\}
\end{array}
$$

$\mathcal{C}_{3}$ is not required in this case.

\footnotetext{
${ }^{3}$ Since $S$ is constant, it does not affect the optimization probelm and it is dropped in the following formulations.
}

- Outermost constellation symbols, the constraints $\mathcal{C}_{1}, \mathcal{C}_{2}$ should guarantee the received signals lie in the correct detection, which is more flexible than the inner constellation points. The constraints can be written as:

$$
\begin{aligned}
\mathcal{C}_{1}: & \mathcal{R}\left\{\mathbf{g}_{j}[n] \mathbf{d}\right\} \geq \sqrt{\gamma_{j}} \mathcal{R}\left\{s_{j}[n]\right\}, \mathcal{R}\left\{s_{j}[n]\right\} \geq 0 \\
& \mathcal{R}\left\{\mathbf{g}_{j}[n] \mathbf{d}\right\} \leq \sqrt{\gamma_{j}} \mathcal{R}\left\{s_{j}[n]\right\}, \mathcal{R}\left\{s_{j}[n]\right\} \leq 0 \\
\mathcal{C}_{2}: & \mathcal{I}\left\{\mathbf{g}_{j}[n] \mathbf{d}\right\} \geq \sqrt{\gamma_{j}} \mathcal{I}\left\{s_{j}[n]\right\}, \mathcal{I}\left\{s_{j}[n]\right\} \geq 0 \\
& \mathcal{I}\left\{\mathbf{g}_{j}[n] \mathbf{d}\right\} \leq \sqrt{\gamma_{j}} \mathcal{I}\left\{s_{j}[n]\right\}, \mathcal{I}\left\{s_{j}[n]\right\} \leq 0 .(11)
\end{aligned}
$$

$\mathcal{C}_{3}$ guarantees that the received symbol has a certain phase. It should be clear that $\tan (\dot{)}$ cannot preserve the sign. Therefore, $\mathcal{C}_{1}, \mathcal{C}_{2}$, and $\mathcal{C}_{3}$ should be used together.

The problem has a quadratic objective and linear constraints, which can be solved efficiently using convex optimization tools [20].

\subsubsection{Detailed Optimization For Rectangular Modulation}

For rectangular modulation (e.g. MQAM), the previous optimization can be simplified as:

$$
\begin{array}{ll}
\mathbf{d} \quad(\mathbf{s}, \mathbf{H}, \mathbf{A}, \boldsymbol{\gamma})=\min _{\mathbf{d}}\left\|\left(\mathbf{I} \otimes \mathbf{A}^{T}\right) \mathbf{d}\right\|^{2}, \\
\text { s.t. } \quad\left\{\begin{array}{l}
\mathcal{C}_{1}: \mathcal{R}\left\{\mathbf{g}_{j}[n] \mathbf{d}\right\} \unlhd \sqrt{\gamma_{j}} \sigma \mathcal{R}\left\{s_{j}[n]\right\} \\
\mathcal{C}_{2}: \mathcal{I}\left\{\mathbf{g}_{j}[n] \mathbf{d}\right\} \unlhd \sqrt{\gamma_{j}} \sigma \mathcal{I}\left\{s_{j}[n]\right\} \forall j \in K, \forall n \in S .
\end{array}\right.
\end{array}
$$

$\mathcal{C}_{1}, \mathcal{C}_{2}$ can be formulated to guarantee that the received signal lies in the correct detection region, which depends on the data symbols. A detailed formulation for $\mathcal{C}_{1}, \mathcal{C}_{2}$ can be expressed as

- For the inner-constellation symbols, $\mathcal{C}_{1}, \mathcal{C}_{2}$ can be formulated as (10).

- Outer constellation symbols, the constraints $\mathcal{C}_{1}, \mathcal{C}_{2}$ should guarantee the received signals lie in the correct detection. The constraints can be written as:

$$
\begin{aligned}
\mathcal{C}_{1}: & \mathcal{R}\left\{\mathbf{g}_{j}[n] \mathbf{d}\right\} \geq \sigma \sqrt{\gamma_{j}} \mathcal{R}\left\{s_{j}[n]\right\}, \mathcal{R}\left\{s_{j}[n]\right\} \geq 0 \\
& \mathcal{R}\left\{\mathbf{g}_{j}[n] \mathbf{d}\right\} \leq \sigma \sqrt{\gamma_{j}} \mathcal{R}\left\{s_{j}[n]\right\}, \mathcal{R}\left\{s_{j}[n]\right\} \leq 0 \\
\mathcal{C}_{2}: & \mathcal{I}\left\{\mathbf{g}_{j}[n] \mathbf{d}\right\}=\sigma \sqrt{\gamma_{j}} \mathcal{I}\left\{s_{j}[n]\right\} . \\
\mathcal{C}_{1}: & \mathcal{R}\left\{\mathbf{g}_{j}[n] \mathbf{d}\right\}=\sigma \sqrt{\gamma_{j}} \mathcal{R}\left\{s_{j}[n]\right\} \\
\mathcal{C}_{2}: & \mathcal{I}\left\{\mathbf{g}_{j}[n] \mathbf{d}\right\} \geq \sigma \sqrt{\gamma_{j}} \mathcal{I}\left\{s_{j}[n]\right\}, \mathcal{I}\left\{s_{j}[n]\right\} \geq 0 \\
& \left.\mathcal{I}\left\{\mathbf{g}_{j}[n] \mathbf{d}\right\} \leq \sigma \sqrt{\gamma_{j}} \mathcal{I}\left\{s_{j}[n]\right\}, \mathcal{I}\left\{s_{j}[n]\right\} \leq \emptyset 14\right)
\end{aligned}
$$

- Outermost constellation symbols, the constraints $\mathcal{C}_{1}, \mathcal{C}_{2}$ should guarantee the received signals lie in the correct detection. The constraints can be formulated as (11).

The problem in (8)-(12) can be solved using linearly constrained quadratic programming [20].

\subsection{Discussion on Receiver Complexity}

In FTN systems, it is required to have an equalizer at receiver to mitigate the effect of ISI [2]. In this work, there is no need for any equalization at the receiver side since ISI is tackled using SLP at the transmitter. Therefore, the receiver architecture is simplified since ISI has been tackled at symbol-level at the transmitter. 


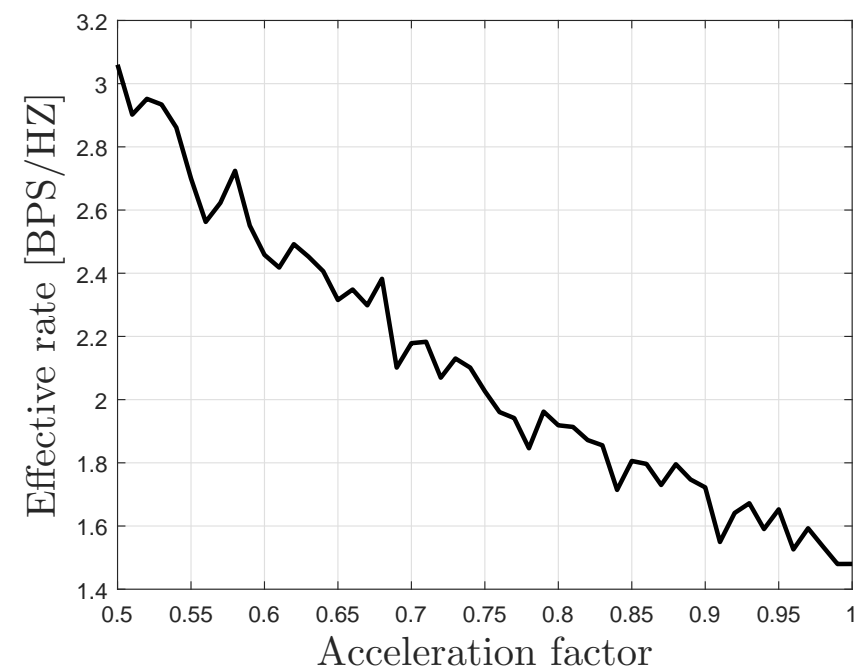

Fig. 2. Effective rate vs acceleration factor

\section{NUMERICAL RESULTS}

In order to assess the performance of the proposed transmissions schemes, Monte-Carlo simulations of the different algorithms have been conducted to study the performance of FTN SLP and compare to spatial domain SLP [12], [13]. The simulated scenario is $K=2$ and each one of them is served using QPSK with $\gamma_{j}=$ $4.77 d B, \forall j \in K, M=10$, the number of channel generation equals to 100 , the channel is fixed for 10 symbols (i.e. $S=10$ ). The adopted channel model is assumed to be

$$
\mathbf{h}_{k} \sim \mathcal{N}\left(0, \sigma^{2}\right)
$$

In this section, we study the performance of the effective rate and energy efficiency at different acceleration factor $\alpha$, it should be highlighted that when $\alpha=1$, the precoding design is the spatial domain SLP as discussed in the literature [12],[13].

Although the rate is usually defined by the assigned modulation, the errors due to the noise effective play an important factor in the achieved rate, which we call it here as effective rate and it is defined as

$$
\bar{R}_{j}=\frac{R_{j}\left(1-S E R_{j}\right)}{\alpha}
$$

where $R_{j}$ is the error -free rate for the user $j$ (the maximum rate can be achieved by a certain modulation). To capture the joint effect of symbol error rate (SER), consumed power and the effective rate, we use the energy efficiency metric, which can be defined as:

$$
\eta=\frac{R_{j}\left(1-S E R_{j}\right)}{\alpha\|\mathbf{D A}\|_{F}^{2}} S
$$

Fig. 2 depicts the effective rate with respect to the acceleration factor $\alpha$. It should be noted that the curve has a decreasing trend with $\alpha$. But this trend is smooth due the fact that sampling at some $\alpha$ leads to better collective properties for all users, for example at some values of $\alpha$ the temporal interference has constructive properties. In $\alpha=0.5-0.51$, the effective rate is higher than $3 \mathrm{bps} / \mathrm{Hz}$. Although the used modulation is QPSK (i.e. maximum rate equals

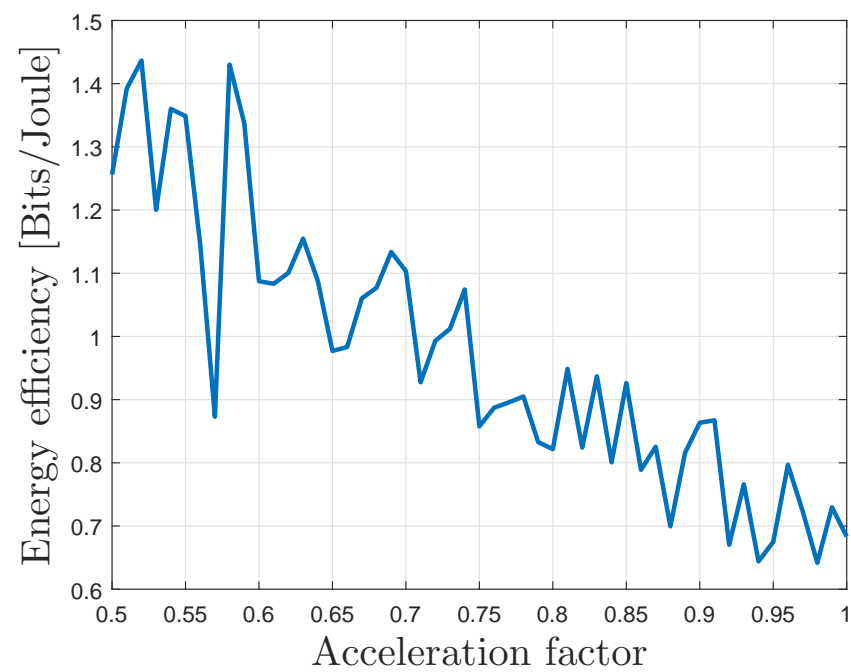

Fig. 3. Energy efficiency vs acceleration factor

to $2 \mathrm{bps} / \mathrm{Hz}$ ), with FTN SLP, we can have effective rate associated with higher modulation (16 APSK, 16 QAM). This effect continues for $\alpha=0.51-0.74$, where the effective rate is associated with (8 PSK, 8QAM) modulations. For $\alpha>0.74$, the effective rate is associated with QPSK modulation. It is interesting finding, we can receive effectively higher rate using less constraints $\mathcal{C}_{1}, \mathcal{C}_{2}$ in (12)(8).

In Fig.3, we depict the performance of the energy efficiency with respect to the accerelation factor. It can be noted that the energy efficiency performance has a different trend than the effective rate due to the fact that the energy efficiency is a function of the both the effective rate and the power required to achieve this target rate so this figure illustrates the tradeoff between them. Furthermore, the general trend is that energy efficiency improves as we accelerate our symbol rate. The highest achieved energy efficiency when $\alpha=0.52$ and $\alpha=0.58$ and the lowest achieved energy efficiency when $\alpha=0.97$ and $\alpha=0.94$, these values are very close to $\alpha=1$. In these cases, the used acceleration cannot compensate for the increased required power to achieve the SNR target.

\section{CONCLUSIONS}

In this paper, we proposed symbol-level precoding scheme for the downlink of multiuser MISO channel based on exploiting FTN. FTN creates intersymbol interference in time domain, which can be treated as additional source of energy to be utilized at the transmitter. Symbol-level processing can exploit the temporal interference within each user stream and the spatial interference among the multiuser streams. FTN SLP achieves impressive gains in terms of effective rate and energy efficiency; it managed to get 2 times improvement in effective rate and energy efficiency in comparison to spatial SLP only.

\section{REFERENCES}

[1] J. Mazo, "Faster-than-Nyquist signaling," Bell System Technical Journal, vol. 54, no. 8, pp. 14511462, 1975. 
[2] J. B. Anderson, F. Rusek, and V. Öwall,"Faster-Than-Nyquist Signaling," Proceedings of the IEEE, Vol. 101, no. 8, pp. 1817 1830, August 2013.

[3] Y. J. D. Kim, J. Bajcsy, and D. Vargas, "Faster-Than-Nyquist Broadcasting in Gaussian Channels: Achievable Rate Regions and Coding," IEEE Transactions on Communications, vol. 64, no. 3, pp. 1016-1030, 2016.

[4] D. Dasalukunte, F. Rusek, and V. Öwall, "Multicarrier fasterthannyquist transceivers: hardware architecture and performance analysis," IEEE Transactions on Circuits and System, vol. 58, no. 4, pp. 827-838, April 2011.

[5] A. Gattami, E. Ringh, and J. Karlsson, “Time Localization and Capacity of Faster-Than-Nyquist Signaling," IEEE Global Communications Conference, San Diego- CA, December 2015.

[6] A. Modenini, F. Rusek, and G. Colavolpe, "Faster-than-Nyquist signaling for next generation communication architectures," $\mathrm{Eu}$ ropean Signal Processing Conference (EUSIPCO), September 2014.

[7] Y. G. Yoo and J. H. Cho, "Asymptotic optimality of binary faster-than-Nyquist signaling," IEEE Communications Letters, vol. 14, no. 9, pp. 788790, Sep. 2010.

[8] F. Rusek, D. Persson, B. K. Lau, E. G. Larsson, T.L. Marzetta, O. Edfors, F. Tufvesson, " Scaling up MIMO: Opportunities and challenges with very large arrays," IEEE Signal Processing Magazine, vol. 30 no. 1, pp. 40-60, 2013.

[9] E. Björnson, E. G. Larsson, T. L. Marzetta, “ Massive MIMO: ten myths and one critical question," IEEE Communications Magazine, vol. 54, no. 2, pp. 114-123, February 2016.

[10] A. Pitarokoilis, S. K. Mohammad, and E. G. Larsson, " On the Optimality of Single-Carrier Transmission in Large-Scale Antenna System,” IEEE Wireless Communications Letters, vol. 1, no. 4, pp. 276-279, 2012.

[11] K. Takeuchi,“ Asymptotic Optimality of Massive MIMO Systems Using Densely Spaced Transmit Antennas," submitted to IEEE Transactions on Information Theory, 2016.

[12] M. Alodeh, S. Chatzinotas and B. Ottersten, "A Multicast Approach for Constructive Interference Precoding in MISO Downlink Channel," in the proceedings of International Symposium in Information theory (ISIT), 2014.

[13] M. Alodeh, S. Chatzinotas and B. Ottersten, "Constructive Multiuser Interference in Symbol Level Precoding for the MISO Downlink Channel," IEEE Transactions on Signal processing, vol. 63, no. 9, pp. 2239 - 2252, May 2015.

[14] C. Masouros and G. Zheng, “Exploiting Known Interference as Green Signal Power for Downlink Beamforming Optimization," IEEE Transactions on Signal processing, vol. 63, , no. 14, pp. 3628 - 3640, July 2015.

[15] M. Alodeh, S. Chatzinotas and B. Ottersten, "Energy Efficient Symbol-Level Precoding in Multiuser MISO Channels Based on Relaxed Detection Region," IEEE Transactions Wireless Communications, vol. 15, no. 5 pp. 3755 - 3767, May 2016.

[16] M. Alodeh, S. Chatzinotas and B. Ottersten, “ Symbol-level Precoding Multiuser MISO Precoding for Multi-level Modulation," IEEE Transactions Wireless Communications, submitted.

[17] D. Spano, M. Alodeh, S. Chatzinotas, and B. Ottersten, "Symbol-Level Precoding for the Non-Linear Multiuser MISO Downlink Channel," in preparation to be submitted to IEEE Transactions on Signal Processing.
[18] A. Kalantari, M. Soltanalian, S. Maleki, S. Chatzinotas and B. Ottersten,"Directional Modulation via Symbol-Level Precoding: A Way to Enhance Security," IEEE Journal in Selected Areas on Signal Processing, to appear.

[19] H. Weingarten, Y. Steinberg, S. Shamai (Shitz), "The Capacity Region of the Gaussian Multiple-Input Multiple-Output Broadcast Channel," IEEE Trans Information Theory, vol. 52 no. 9, pp. 3936-3964, 2006

[20] S. Boyd and L. Vandenberghe, Convex Optimization, Cambridge University Press. 